\title{
A bibliometric analysis and visualization of photothermal therapy on cancer
}

\author{
Xiaoyan Wang ${ }^{1 \#}$, Dan $\mathrm{Li}^{2 \#}$, Xinhe Huang ${ }^{3}$, Qi $\mathrm{Luo}^{4}$, Xue $\mathrm{Li}^{5}$, Xianqin Zhang ${ }^{6}$, Lin Zhang ${ }^{7,8}$ \\ ${ }^{1}$ Department of Pathology, Clinical Medical College and The First Affiliated Hospital of Chengdu Medical College, Chengdu, China; ${ }^{2}$ School \\ of Laboratory Medicine, Chengdu Medical College, Chengdu, China; ${ }^{3}$ School of Life Science and Engineering, Southwest Jiaotong University, \\ Chengdu, China; ${ }^{4}$ School of Biomedical Sciences, Chengdu Medical College, Chengdu, China; ${ }^{5}$ School of Pharmacy, Chengdu Medical College, \\ Chengdu, China; ${ }^{6}$ School of Basic Medical Sciences, Chengdu Medical College, Chengdu, China; ${ }^{7}$ College of Pharmaceutical Sciences, Zhejiang \\ Chinese Medical University, Hangzhou, China; ${ }^{8}$ Department of Pharmacy, Shaoxing People's Hospital, Zhejiang University School of Medicine, \\ Shaoxing, China \\ Contributions: (I) Conception and design: L Zhang; (II) Administrative support: None; (III) Provision of study materials or patients: None; (IV) \\ Collection and assembly of data: X Wang, D Li; (V) Data analysis and interpretation: Q Luo, X Li, X Zhang; (VI) Manuscript writing: All authors; (VII) \\ Final approval of manuscript: All authors. \\ \#These authors contributed equally to this work. \\ Correspondence to: Lin Zhang, PhD. Professor, College of Pharmaceutical Sciences, Zhejiang Chinese Medical University, Hangzhou 310053 , China. \\ Email: zhanglinfudan@zju.edu.cn.
}

Background: Cancer is one of the most lethal diseases in the world, and photothermal therapy was reported recently as a new and effective therapy for cancer. This study offers the bibliometric and visualization analysis of photothermal therapy on cancer.

Methods: A record of 6,233 papers in this field from 1995 to 2019 was obtained based on the Web of Science Core Collection (WoSCC). And CiteSpace was used to analyze the annual trends of publications, countries, institutions, journals, co-cited references, and keywords in the field of photothermal therapy on cancer.

Results: We identified that the number of publications continually increased over the time. The most productive country and institution in this field was China and Chinese Academy of Sciences, respectively. The ACS Appl Mater Interfaces was the most active journal. Co-cited references analysis revealed the top landmark articles in the field. Co-occurrence keywords and their clustered network were analyzed, revealing that materials, especially nanomaterials, used in photothermal therapy, remained the hotspots in this research field. Timezone view and burst detection of keywords showed that nanomaterials were always the hotspots and the frontier topics in this field.

Conclusions: The current study revealed that photothermal therapy has become a subject of growing study and a very important research area. In addition, the research of materials in photothermal therapy, especially nanomaterials, which were applied in photothermal therapy to treat cancer effectively, is the foci and the frontier topic in this field.

Keywords: Photothermal therapy; cancer; CiteSpace; visualization analysis; nanomaterials

Submitted Sep 29, 2020. Accepted for publication Jan 29, 2021.

doi: $10.21037 /$ tcr-20-2961

View this article at: http://dx.doi.org/10.21037/tcr-20-2961

\section{Introduction}

Cancer overtook heart disease and became an important reason of death all over the world $(1,2)$. It was estimated that there were 18.1 million new cancer cases and 9.6 million deaths of cancer, according to the International Agency for Research on Cancer (IARC) estimated in 2018 (3). On the other side, surgery, radiotherapy, and chemotherapy are the main ways to treat clinical cancer at present. Unfortunately, 


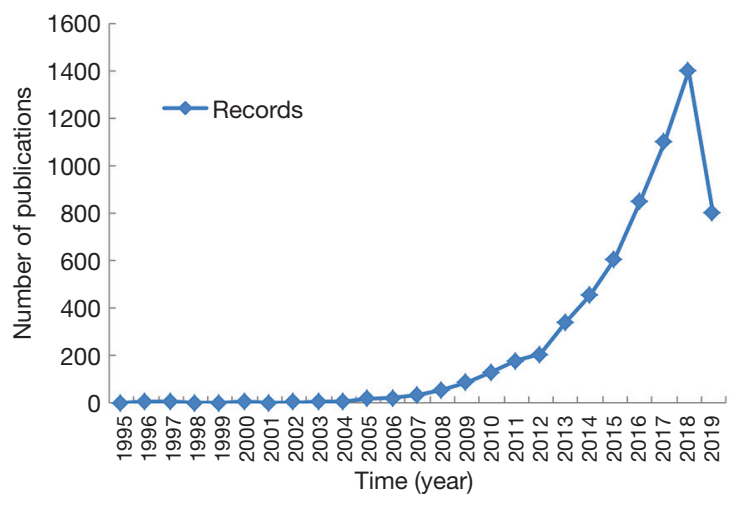

Figure 1 The annual trends of publications of photothermal therapy on cancer.

patients have to suffer the drawbacks of these tradition treatments, such as normal cells killed, the immune system destroyed, and the risk of a second cancer increased (4-6). Photothermal therapy is becoming a better treatment on cancer due to its high targeting and effectiveness (7). Both photothermal therapeutic (PTT) agent accumulation and localized near-infrared (NIR) laser irradiation were used in photothermal therapy, making its therapeutic effects occur only at the sites of the tumor, therefore it can avoid the drawbacks of traditional treatments mentioned above. Compared with traditional techniques, photothermal therapy has many other advantages including simple operation, fewer complications, quicker recovery, and shorter length of stay in hospital (8).

Meanwhile, nanotechnology is extensively explored for drug delivery. Nanocarrier delivery offered a great opportunity to improve the bioactivity of drugs and the biocompatibility of tumor therapy (9-11). Nanomaterials, such as nanoparticles, nanoshell, and nanorods, had strong near-infrared absorption ability. Hence, they are able to convert near-infrared laser into heat effectively by photothermal process. Recently, nanomaterials have become the hot spot in photothermal therapy, especially in designing new nanoparticle-drug conjugates, which produce the photothermally activated physical and biological effects, to treat cancer effectively (12-16).

In this study, the quantity and quality of global publications of photothermal therapy on cancer from 1995 to 2019 were assessed with bibliometric analysis. The objective of our research was to study and analyze the global scientific outputs of photothermal therapy on cancer research and to estimate the research trends and hotspots of this field. We found that the continuous advances of this field and application of novel nanomaterials would enhance the future development of cancer photothermal therapy.

\section{Methods}

\section{Data acquisition}

A record of 6,233 papers published between 1995 and 2019 from the Web of Science Core Collection (WoSCC) was obtained. The WoSCC is the most frequently used source of scientific information. We used the term \# 1 (TS= "photothermal" and "cancer") or \# 2 (TS= "photothermal" and "tumor") to search the related articles, and removed the duplicates. The final record was obtained on July 23, 2019.

\section{Statistical analysis}

We used the WoSCC and CiteSpace V 5.0R1 SE to analyze the annual trends of publications, countries, institutions, cocited articles, and keywords in cancer photothermal therapy field $(17,18)$. The parameters of CiteSpace were as follows: time slicing [1995-2019], years per slice (1), term source (all selection), node type (choose one at a time), and pruning (pathfinder, Pruning the merged network, Pruning Sliced Networks).

\section{Results}

\section{The annual trends of publications of photothermal therapy on cancer}

The web of science collection provided 5,866 documents with topic words of "photothermal and cancer" in different format of publications and provided 2,033 files with the topic words of "photothermal and tumor". After removing the duplicates, 6,233 publications were obtained. As shown in Figure 1, the number of published articles of photothermal therapy on cancer increased steadily from 1995 to 2010, and the number of publications increased rapidly from 2,011 onwards. From 2017 to 2018, the activity of photothermal therapy on cancer reached a peak.

\section{The distribution of countries and institutions}

We analyzed the distribution of countries in the research field of photothermal therapy on cancer. There were 72 different countries ranked. Among them, the top 10 countries were China, USA, South Korea, India, Singapore, 


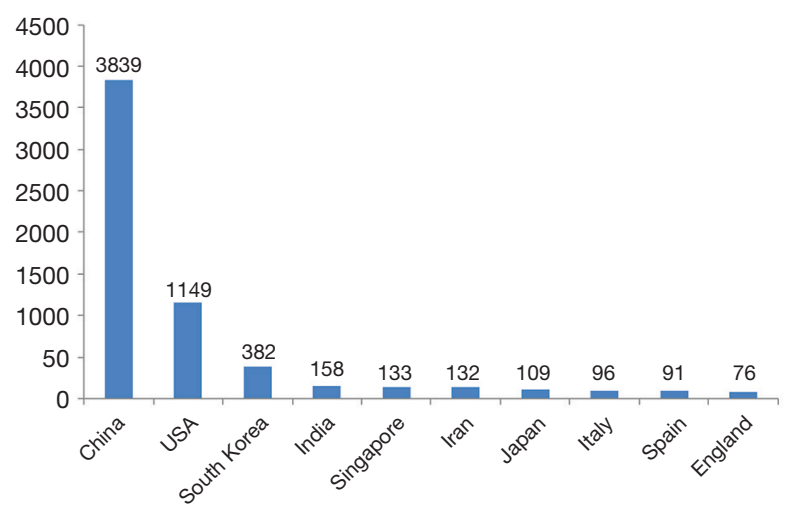

Figure 2 The top 10 countries of publications of photothermal therapy on cancer.

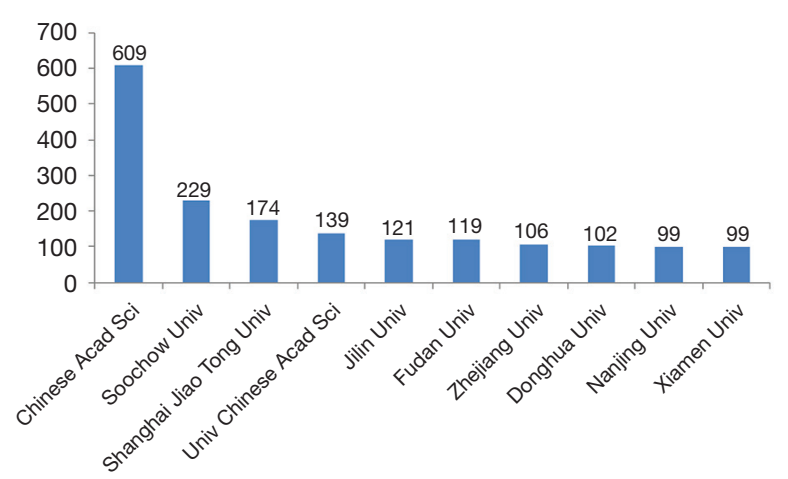

Figure 3 The top 10 institutes of publications of photothermal therapy on cancer.
Iran, Japan, Italy, Spain, and England (Figure 2). The results showed that china was the country with the largest number of publications in this field.

Figure 3 showed the top 10 institutions among 277 institutions in the world, which published the largest number of articles of photothermal therapy on cancer. Chinese Academy of Sciences (Chinese Acad Sci) published the most papers, with 609 papers, accounting for $9.63 \%$ of the total published papers in this field. The second one was the Soochow University with 229 publications, followed by Shanghai Jiao Tong University [174], the University Chinese Academy of Sciences [139], Jilin University [121], Fudan University [119], Zhejiang University [106], Donghua University [102], Nanjing University [99] and Xiamen University [99]. Most interestingly, all top 10 institutes were from China, consistent with the statistical analysis of national publication of photothermic therapy on cancer.

\section{Analysis of journals of publications of photothermal therapy on cancer}

A total of 112 journals published 6,233 articles of photothermal therapy on cancer. The characteristics of the top 10 active journals were shown in Table 1. Among the top 10 publishers of the journals, three were from American, three were from British, two were from German journals, one was Netherlands journal and one was New Zealanian journal. The largest number of articles in this area was

Table 1 The top 10 journals of publications on photothermal therapy for cancer

\begin{tabular}{llcccc}
\hline Rank & Journal & Frequency & Country & JCR & Impact factor [2018] \\
\hline 1 & ACS Appl Mater Interfaces & 417 & USA & Q1 & 8.456 \\
2 & Nanoscale & 299 & England & Q1 & 6.97 \\
3 & Biomaterials & 290 & Netherlands & Q1 & 10.273 \\
4 & J Mater Chem B & 281 & England & Q2 & 5.047 \\
5 & ACS Nano & 242 & USA & Q1 & 13.903 \\
6 & RSC Advances & 176 & England & Q3 & 3.049 \\
7 & Small & 152 & Germany & Q1 & 10.856 \\
8 & Adv Funct Mater & 149 & Germany & Q1 & 15.621 \\
9 & Adv Mater & 137 & USA & Q1 & 25.809 \\
10 & Int J Nanomedicine & 137 & New Zealand & Q2 & 4.471 \\
\hline
\end{tabular}

JCR, journal citation reports. 
published on ACS Applied Materials and Interfaces, followed by Nanoscale and Biomaterials. The journal with highest impact factor was Adv Mater, followed by Adv Funct Mater.

\section{Analysis of the co-cited references}

The clustered network consists of those references with higher centrality and citation counts was shown in Figure 4. Visualization of co-cited references showed a total of 646 nodes and 1,578 links. Each node represents a cited article. The link lines between the nodes represent the relationships between the articles. The area of each node was proportional to the total co-citation frequency of the associated publications.

Table 2 showed the top 10 co-cited references, including their authors, year, journal, cited frequency and title. The author Robinson (19) published in 7 Am Chem Soc had the highest number of citations (611 citations), followed by Yang (20) in Nano Lett (604 citations), and Cheng (9) in Chem Rev (595 citations). These ten references were often considered fundamental in the research field of photothermal therapy on cancer.

\section{Analysis of co-occurrence keywords and burst keywords}

Keywords co-occurrence can effectively reflect the research hotspots in the field, and burst keywords could indicate frontier topics (27). To explore research hotspots and frontier topics, we analyzed the distribution of keywords. The keywords co-occurrence network map, the top 10 keywords, clustered network of co-occurrence keywords, the keywords timezone view, and keywords with the strongest citation bursts of publications in this field were presented.

The keyword co-occurrence network of publications of photothermal therapy on cancer was constructed with 6,233 articles (Figure 5). The nodes in the map represented the corresponding keywords, the node size represented the number of publications in the field of photothermal therapy that include the keywords. The link lines between the nodes represented the relationships between the keywords. For example, a great many of connection lines were observed between cancer and other keywords nodes, indicating that the keyword "cancer" has a close relationship with other keywords in this field. From these keywords, we obtained the top 10 co-occurrence keywords (Table 3) according to their frequency occurred in the publications. The hot keywords were photothermal therapy, nanoparticle, drug delivery, in vivo, cancer, cancer therapy, gold nanoparticle, photodynamic therapy, cell, and therapy.
We mapped 6,233 publications of photothermal therapy on cancer via a clustered network in hierarchical order (Figure 6). Different co-occurrence keywords were represented by the nodes and a distinct specialty or a thematic concentration was represented by the clusters. The co-occurrence keywords knowledge map revealed keywords with higher centrality and occurrence counts. The area of each node was proportional to the total co-occurrence frequency of the associated reference. The co-occurrence keywords could be clustered into eleven main sub-clusters including \#0discrete particle model, \#1liposome, \#2optical property, \#3nanostructure, \#4optical property, \#5performance liquid chromatography, \#6laser immunotherapy, \#7nanostructure, \#8reduced graphene oxide, \#9multifunctional germanium nanoparticle, and \#10near infrared responsive.

Figure 7 displayed a timezone view of co-occurrence keywords. From Figure 7 we can demonstrated that, before 2000 , the research of photothermal therapy on cancer was little and the keywords in that time were concentrated on "tumor", "photodynamic therapy" and "cancer", etc. Since 2000, the research in this field began to increase, and the field of key words became extensive, such as "photothermal therapy", "in vivo", "cell", "nanopaticle" and "drug delivery", etc. Since 2010, the publications of photothermal therapy increased rapidly. Many new nanomaterials, like "nanomaterial graphene", "mesoporous silica nanoparticle" and "iron oxide nanopaticle", were employed in photothermal therapy. The link between these keywords in Figure 7 was concentrated and progressive, revealing the close connection of these keywords in the field.

So-called "burst words" represent words that are cited frequently over a period of time. CiteSpace was used to detect burst keywords, which were considered to be indicators of research frontier topics over time (27). In this study, we detected bursts from 1995 to 2019 based on analysis of 6,233 original articles. The timeline was depicted as a blue line, and the time interval that a subject was found to have a burst was shown as a red segment on the blue timeline. It indicated the beginning year, the ending year, and the duration of the burst. Among the top 65 keywords with the highest burst strength, we were particularly interested in those keywords with research significance which indicated the evolution trend of the photothermal therapy research field (Table 4). The keywords with more stronger citation bursts in photothermal therapy articles were laser [1997-2013], optical property [2005-2014], gold nanoparticle [2006-2014], nanoshell [2006-2014], size [2007-2011], metal nanoparticle [2007-2013], shape 


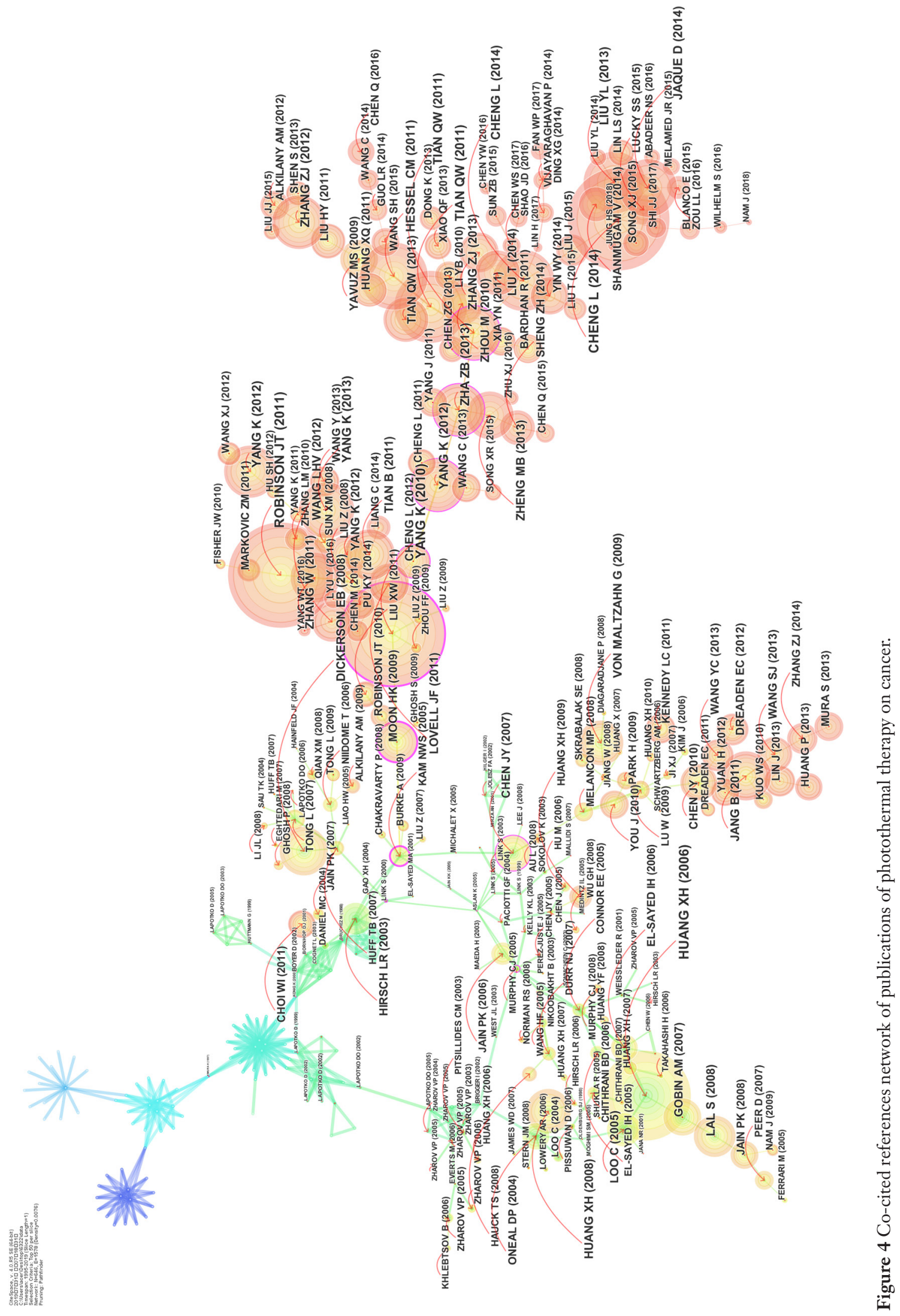


Table 2 The top 10 co-cited references of photothermal therapy on cancer

\begin{tabular}{|c|c|c|c|c|}
\hline Authors & Years & Journal & Cited frequency & Title \\
\hline Cheng (9) & 2014 & Chem Rev & 595 & Functional nanomaterials for phototherapies of cancer \\
\hline Tian (22) & 2011 & ACS Nano & 426 & $\begin{array}{l}\text { Hydrophilic Cu9S5 nanocrystals: a photothermal agent with a } 25.7 \% \\
\text { heat conversion efficiency for photothermal ablation of cancer cells in } \\
\text { vivo }\end{array}$ \\
\hline Hessel (23) & 2011 & Nano Lett & 397 & Copper selenide nanocrystals for photothermal therapy \\
\hline Cheng (25) & 2014 & Adv Mater & 365 & $\begin{array}{l}\text { PEGylated WS (2) nanosheets as a multifunctional theranostic agent } \\
\text { for in vivo dual-modal CT/photoacoustic imaging guided photothermal } \\
\text { therapy }\end{array}$ \\
\hline Jaque (10) & 2014 & Nanoscale & 335 & Nanoparticles for photothermal therapies \\
\hline Yang (26) & 2013 & Chem Soc Rev & 323 & Nano-graphene in biomedicine: theranostic applications \\
\hline
\end{tabular}

[2007-2014], nanorod [2008-2012], tumor cell [2009-2012], photothermal cancer therapy [2009-2013], nanomaterial grapheme [2009-2014], nanocage [2010-2014], multifunctional nanoparticle [2010-2014]. Since 2015, many researches in cancer photothermal therapy filed have focused on conversion efficiency [2015-2016] and guided photothermal therapy [2015-2019].

\section{Discussion}

In the present study, we used information visualization methods to analyze the publications of photothermal therapy on cancer between 1995 and 2019. The annual trends of publications of photothermal therapy on cancer (Figure 1) showed that there were more and more scientific research publications over the past $20+$ years. Of note, publications increased rapidly after 2010. Before 2010, there were only 381 publications collectively in this field, accounting for $6.11 \%$ of the total number of publications. The reason of the above results was that the doctors firstly observed the beneficial effects of thermal therapy for the treatment of cancer in the 19th century (28). And photothermal therapy was firstly reported by professor Halas from Rice University in 2003 , as a new therapy on cancer (29). We found that photothermal therapy has become a subject of growing study and an increasingly important area of cancer research.

Secondly, we investigated the distribution of countries and institutions in this field. Based on the top 10 countries and institutes of publications of photothermal therapy on cancer (Figures 2,3), we identified that China contributed most to the number of articles on photothermal therapy and China was in a leading position in this field, followed by USA. The incidence and mortality of cancer have been increased rapidly year after year. Cancer incidence and mortality in China were the highest compared with other countries in the world. Therefore, it has made cancer the leading cause of death since 2010 and a major public health problem in China $(3,30)$. From then on, China has to face huge challenges in managing the large and increasing burden of cancer. And China urgently requires concerted efforts and commitment from all levels of government and nongovernment organizations. Thus, more and more Chinese researchers focused on the cancer and cancer treatment research area.

Thirdly, the top 10 journals which published the greatest number of articles of photothermal therapy on cancer (Table 1) were mostly journals in material science and bioengineering, such as ACS Appl Mater Interfaces, Nanoscale, 


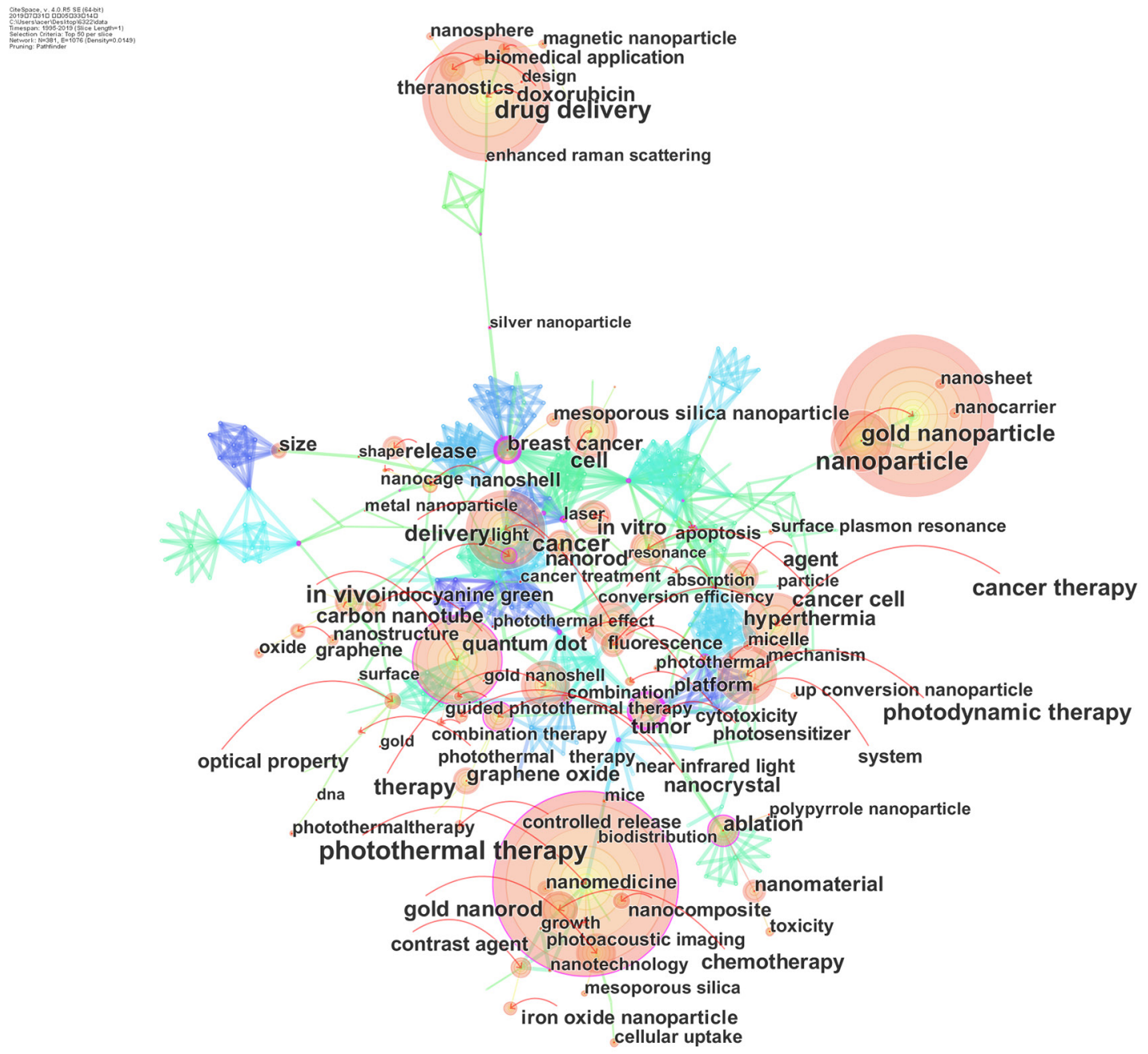

Figure 5 Co-occurrence keywords network of publications of photothermal therapy on cancer.

Table 3 The top 10 keywords of publications of photothermal therapy on cancer

\begin{tabular}{lccc}
\hline Rank & Keywords & Frequency & Centrality \\
\hline 1 & Photothermal therapy & 2,488 & 0.15 \\
2 & Nanoparticle & 2,193 & 0.02 \\
3 & Drug delivery & 1,760 & 0.07 \\
4 & In vivo & 1,241 & 0.13 \\
5 & Cancer & 1,127 & 0.06 \\
6 & Cancer therapy & 954 & 0 \\
7 & Gold nanoparticle & 903 & 0.05 \\
9 & Photodynamic therapy & 887 & 0.02 \\
10 & Cell & 764 & 0.02 \\
\hline
\end{tabular}




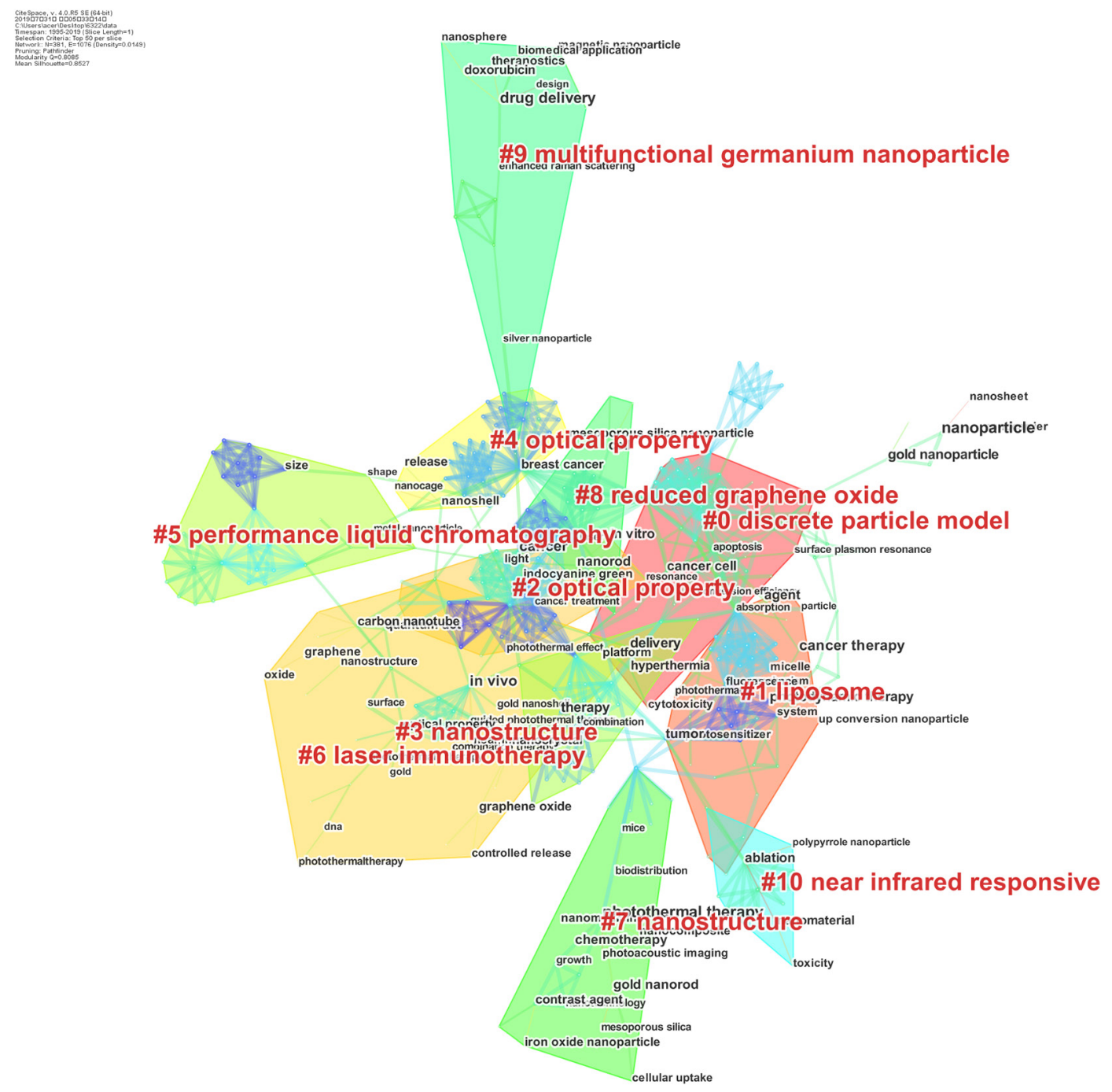

Figure 6 Co-occurrence clustered keywords network of publications of photothermal therapy on cancer.

Biomaterials, 7 Mater Chem B and ACS Nano. This revealed that research in materials, particularly the nanophase materials, became a central topic of this field. The impact factors [2018] of these journals were relatively high, and most journals were classified as Q1 by journal citation reports (JCR), indicating these journals had a significant influence in this research field. The articles published in these journals could lead the trend of this research field. Therefore, new materials involved in photothermal therapy on cancer should lead to promising results of cancer treatment.

Fourthly, we analyzed the co-cited references. Among the top 10 co-cited references in 2006, Huang et al. (21) reported that gold nanorods was employed for cancer cell imaging and photothermal therapy materials in the nearinfrared region. Then they found that after exposure to continuous red laser at $800 \mathrm{~nm}$ from gold nanorods, cancer cells require about half the laser energy to be photothermally destroyed than the nonmalignant cells. In 2010, Yang et al. (20) studied the in vivo behaviors of nanographene sheets (NGS) with polyethylene glycol (PEG) coating by a fluorescent labeling method, and they successfully used this carbon nanomaterial in photothermal therapy in vivo by intravenous administration, which was the first successful case in the world. Subsequently, Robinson et al. (19) reported a new nanosized material for potential photothermal therapy, which reduced graphene oxide sheeted with NIR light absorbance 


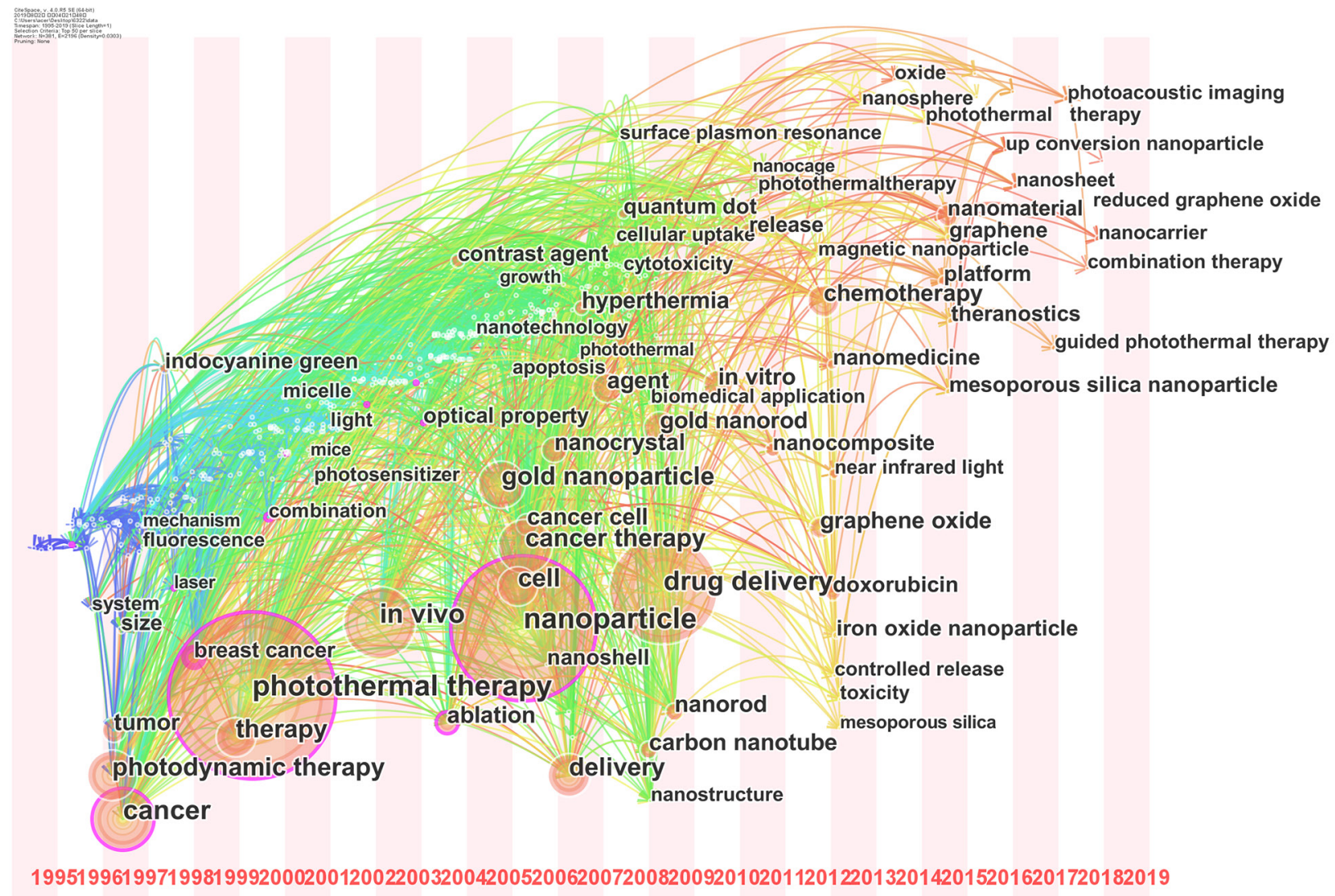

Figure 7 Co-occurrence keywords timezone view of publications of photothermal therapy on cancer.

Table 4 Keywords with more stronger citation bursts in articles of photothermal therapy on cancer published during 1995-2019

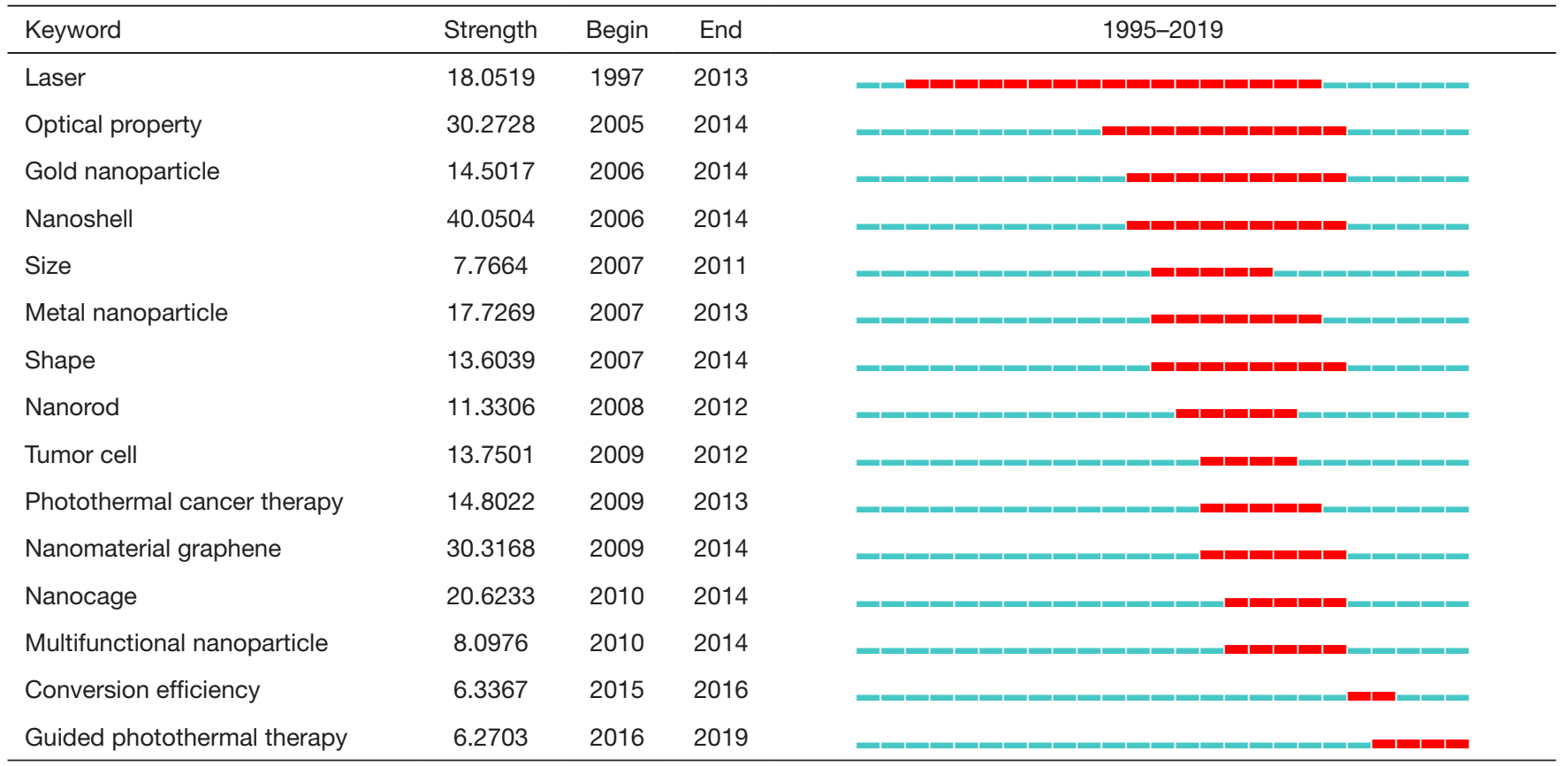


and biocompatibility in 2011. Almost at the same time, Hessel et al. (23) found that $\mathrm{Cu}(2-\mathrm{x}) \mathrm{Se}$ nanocrystals as an photothermal therapy materials, could lead to the human colorectal cancer cell (HCT-116) destruction. And in November, 2011, Tian et al. (22) developed hydrophilic plate-like $\mathrm{Cu}_{(9)} \mathrm{S}_{(5)}$ nanocrystals as a new photothermal agent, and demonstrated that under the irradiation of 980 $\mathrm{nm}$ laser with the conservative and safe power density over a short period, the photothermal effects of the $\mathrm{Cu}_{(9)} \mathrm{S}_{(5)}$ NCs could efficiently kill cancer cells in vivo. After that, Liu et al. (24) investigated the dopamine-melanin colloidal nanospheres, as efficient near-infrared PTT materials for in vivo cancer treatment in 2013. Recently, Cheng et al. (25) developed a new photothermal theranostic agents, PEGylated WS2 nanosheets, which could completely ablate the tumors in 2014.

The remaining 3 co-cited references are reviews. Among them, two articles reviewed the present status of the use of nanoparticles for photothermal therapy in details, the advantages and disadvantages of the different heating nanoparticles were discussed $(9,10)$. And the last one focused on nano-graphene in photothermal treatment, summarizing the latest progress in this rapidly growing field, discussing the future prospects and challenges of using graphene-based materials for theranostic applications (26). The above results suggested that the applications of nanomaterials in photothermal therapy, for example gold nanorods, nano-graphene and $\mathrm{Cu}(2-\mathrm{x})$ Se nanocrystals, etc., was burst and hot in this research field, all of which are identical with those conclusions of analyzing the journals.

Lastly, but most importantly, we analyzed the distribution of keywords in the field of photothermal therapy on cancer. To reflect hot topics, the high frequency co-occurrence keywords (Figure 5 and Table 3) and co-occurrence keywords clustered network (Figure 6) were analyzed. The results suggested that photothermal therapy as a new effective cancer treatment, its materials, especially nanomaterials, remained the hotspots in this research field. To explore frontier topics, we studied the co-occurrence keywords timezone view (Figure 7) and the keywords with more stronger citation bursts (Table 4). Photothermal therapy, in which nanomaterials embedded within tumors generate heat in response to exogenously applied laser light, has been well documented as an independent strategy for highly selective cancer treatment (31-33). Therefore, nanomaterials are always the hotspots and the frontier topics in this field. Different nanomaterials, like gold nanoparticle, nanographene and $\mathrm{Cu}(2-\mathrm{x})$ Se nanocrystals, etc, are widely used in this field, including in the diagnosis, monitoring, and treatment of diseases. The shapes of the nanomaterials are varied, showing as nanoshell, nanoparticles, nanorub, nanocrystal and nanotube. And different shapes showed different properties in physical and chemical. Overall, these characteristics could make benefits to the photothermal therapy on cancer (34-36).

"Gold nanoparticle" was the keyword with strongest citation bursts. Gold nanoparticles were the main agent used in photothermal therapy because the following reasons. First, they have good biocompatibility and efficient light-to-heat conversion. Next, they are tuned to absorb near-infrared light, which penetrates tissue more deeply than other wavelengths of light. Again, they offer simple gold-thiol bioconjugation chemistry for the attachment of desired molecules. Finally, they exist small diameters that enable tumor penetration upon systemic delivery $(31,37,38)$.

Another keyword was the nanomaterial graphene, which is a hot study in photothermal therapy. Graphene and its derivatives exhibit unique physical and chemical properties, hence they are useful vehicles for photothermal therapy-based cancer treatment, including enhancement of multimodalities, guided imaging, enhanced chemotherapy and low-power efficient photothermal therapy for optimum therapeutic efficiency. Graphene-based nanomaterials, with NIR absorption properties, can provide successful thermal ablation in cancer cells. In the recent years, advances in graphene-based photothermal therapy have produced efficient and efficacious tumor inhibition via nanomaterial structural design and different functionalization of graphene-derived nanocomposites (19,39-41).

Of note, we found that photothermal therapy is mostly applied in epidermic tumors, such as breast cancer and skin tumors, due to the poor penetration ability of the infrared light. As a result, there is a lack of researches for treatment of solid tumors in the deep layers of the body using photothermal therapy, which needs to be deeply studied in the future.

From 2015 onwards, the investigation of carrier materials to guide photothermal therapy and the development of materials were concerned, by analyzing the keywords "guided photothermal therapy" and "conversion efficiency". Conversing more efficiency from exogenously applied laser light have been become the frontier topics in this field. We predict the photothermal therapy will be frequently employed in cancer treatment, not only for treating superficial tumors, such as breast cancer, but also for treating other solid tumor. Researchers should pay more attention on surmounting the defects of current photothermal therapy, developing better 
materials applied in photothermal therapy to treat cancers.

\section{Conclusions}

There is no doubt that photothermal therapy on cancer has significantly advanced over the past $20+$ years. With the help of information visualization, we were able to identify research foci and overall trends in the field. The results of our study could offer gathered information for future research. The development of better materials, applied in photothermal therapy to destroy cancer cells targetingly and effectively, is always the foci in this research field in present and in future. However, the side effects and cell toxicity of the materials should be pay more attention when using the materials in humans $(15,16)$.

\section{Acknowledgments}

Funding: This work was supported by Opening project of Zhejiang provincial preponderant and characteristic subject of key university (traditional Chinese pharmacology), Zhejiang Chinese Medical University (No. ZYAOX2018035), the Project of Shaoxing Medical Key Discipline Construction Plan (No. 2019SZD06), Natural Science Foundation of Sichuan Science and Technology Department (No. 2019YJ0582), and the Key R\&D Projects of Sichuan Province, China (No. 2018SZ0061).

\section{Footnote}

Conflicts of Interest: All authors have completed the ICMJE uniform disclosure form (available at http://dx.doi. org/10.21037/tcr-20-2961). The authors have no conflicts of interest to declare.

Ethical Statement: The authors are accountable for all aspects of the work in ensuring that questions related to the accuracy or integrity of any part of the work are appropriately investigated and resolved.

Open Access Statement: This is an Open Access article distributed in accordance with the Creative Commons Attribution-NonCommercial-NoDerivs 4.0 International License (CC BY-NC-ND 4.0), which permits the noncommercial replication and distribution of the article with the strict proviso that no changes or edits are made and the original work is properly cited (including links to both the formal publication through the relevant DOI and the license).
See: https://creativecommons.org/licenses/by-nc-nd/4.0/.

\section{References}

1. Yoo D, Lee JH, Shin TH, et al. Theranostic magnetic nanoparticles. Acc Chem Res 2011;44:863-74.

2. Bardhan R, Lal S, Joshi A, et al. Theranostic nanoshells: from probe design to imaging and treatment of cancer. Acc Chem Res 2011;44:936-46.

3. Bray F, Ferlay J, Soerjomataram I, et al. Global cancer statistics 2018: GLOBOCAN estimates of incidence and mortality worldwide for 36 cancers in 185 countries. CA Cancer J Clin 2018;68:394-424.

4. Kievit FM Zhang M. Surface engineering of iron oxide nanoparticles for targeted cancer therapy. Acc Chem Res 2011;44:853-62.

5. Vogel A Venugopalan V. Mechanisms of pulsed laser ablation of biological tissues. Chem Rev 2003;103:577-644.

6. Nolsøe CP, Torp-Pedersen S, Burcharth F, et al. Interstitial hyperthermia of colorectal liver metastases with a USguided Nd-YAG laser with a diffuser tip: a pilot clinical study. Radiology 1993;187:333-7.

7. Lal S, Clare SE, Halas NJ. Nanoshell-enabled photothermal cancer therapy: impending clini cal impact. Acc Chem Res 2008;41:1842-51.

8. Dreaden EC, Mackey MA, Huang X, et al. Beating cancer in multiple ways using nanogold. Chem Soc Rev 2011;40:3391-404.

9. Cheng L, Wang C, Feng L, et al. Functional nanomaterials for phototherapies of cancer. Chem Rev 2014;114:10869-939.

10. Jaque D, Martinez Maestro L, del Rosal B, et al. Nanoparticles for photothermal therapies. Nanoscale 2014;6:9494-530.

11. Yavuz MS, Cheng Y, Chen J, et al. Gold nanocages covered by smart polymers for controlled release with near-infrared light. Nat Mater 2009;8:935-9.

12. Wang Y, Black KC, Luehmann H, et al. Comparison study of gold nanohexapods, nanorods, and nanocages for photothermal cancer treatment. ACS Nano 2013;7:2068-77.

13. Tchounwou C, Sinha SS, Viraka Nellore BP, et al. Hybrid Theranostic Platform for Second Near-IR Window Light Triggered Selective Two-Photon Imaging and Photothermal Killing of Targeted Melanoma Cells. ACS Appl Mater Interfaces 2015;7:20649-56.

14. Perrault SD, Walkey C, Jennings T, et al. Mediating tumor targeting efficiency of nanoparti-cles through design. Nano Lett 2009;9:1909-15. 
15. Siddique S, Chow JCL. Application of Nanomaterials in Biomedical Imaging and Cancer Therapy. Nanomaterials 2020;10:1700.

16. Siddique S, Chow JCL. Gold Nanoparticles for Drug Delivery and Cancer Therapy. App Sci 2020;10:3824.

17. Chen C. CiteSpace II: detecting and visualizing emerging trends and transient patterns in scientific literature. J Am Soc Inf Sci Technol 2006;57:359-77.

18. Chen $\mathrm{C}, \mathrm{Hu} \mathrm{Z}$, Liu S, et al. Emerging trends in regenerativemedicine: a scientometric analysis in CiteSpace. Exp Opin Biol Ther. 2012;12:593-608.

19. Robinson JT, Tabakman SM, Liang Y, et al. Ultrasmall reduced graphene oxide with high near-infrared absorbance for photothermal therapy. J Am Chem Soc 2011;133:6825-31.

20. Yang K, Zhang S, Zhang G, et al. Graphene in mice: ultrahigh in vivo tumor uptake and effi-cient photothermal therapy. Nano Lett 2010;10:3318-23.

21. Huang X, El-Sayed IH, Qian W, et al. Cancer cell imaging and photothermal therapy in the near-infrared region by using gold nanorods. J Am Chem Soc 2006;128:2115-20.

22. Tian Q, Jiang F, Zou R, et al. Hydrophilic Cu9S5 nanocrystals: a photothermal agent with a $25.7 \%$ heat conversion efficiency for photothermal ablation of cancer cells in vivo. ACS Nano 2011;5:9761-71.

23. Hessel CM, Pattani VP, Rasch M, et al. Copper selenide nanocrystals for photothermal ther-apy. Nano Lett 2011;11:2560-6.

24. Liu Y, Ai K, Liu J, et al. Dopamine-melanin colloidal nanospheres: an efficient near-infrared photothermal therapeutic agent for in vivo cancer therapy. Adv Mater 2013;25:1353-9.

25. Cheng L, Liu J, Gu X, et al. PEGylated WS(2) nanosheets as a multifunctional theranostic agent for in vivo dualmodal CT/photoacoustic imaging guided photothermal therapy. Adv Mater 2014;26:1886-93.

26. Yang K, Feng L, Shi X, et al. Nano-graphene in biomedicine: theranostic applications. Chem Soc Rev 2013;42:530-47.

27. Liang YD, Li Y, Zhao J, et al. Study of acupuncture for low back pain in recent 20 years: a bib-liometric analysis via CiteSpace. J Pain Res 2017;10:951-64.

28. Habash RW, Bansal R, Krewski D, et al. Thermal therapy, part 2: hyperthermia techniques. Crit Rev Biomed Eng 2006;34:491-542.

29. Hirsch LR, Stafford RJ, Bankson JA, et al. Nanoshellmediated near-infrared thermal therapy of tumors under magnetic resonance guidance. Proc Natl Acad Sci U S A 2003;100:13549-54.

30. Chen $W$, Zheng R, Baade PD, et al. Cancer statistics in China, 2015. CA Cancer J Clin 2016;66:115-32.

31. Kennedy LC, Bickford LR, Lewinski NA, et al. A new era for cancer treatment: gold-nanoparticle-mediated thermal therapies. Small 2011;7:169-83.

32. Guo L, Yan DD, Yang D, et al. Combinatorial photothermal and immuno cancer therapy using chitosancoated hollow copper sulfide nanoparticles. ACS Nano 2014;8:5670-81.

33. Hashida Y, Tanaka H, Zhou S, et al. Photothermal ablation of tumor cells using a sin-gle-walled carbon nanotubepeptide composite. J Control Release 2014;173:59-66.

34. Chithrani BD, Ghazani AA, Chan WC. Determining the size and shape dependence of gold nanoparticle uptake into mammalian cells. Nano Lett 2006;6:662-8.

35. Chithrani BD Chan WC. Elucidating the mechanism of cellular uptake and removal of pro-tein-coated gold nanoparticles of different sizes and shapes. Nano Lett 2007;7:1542-50.

36. Osaki F, Kanamori T, Sando S, et al. A quantum dot conjugated sugar ball and its cellular up-take. On the size effects of endocytosis in the subviral region. J Am Chem Soc 2004; 126:6520-1.

37. Riley RS Day ES. Gold nanoparticle-mediated photothermal therapy: applications and op-portunities for multimodal cancer treatment. Wiley Interdiscip Rev Nanomed Nanobi-otechnol 2017;9:10.

38. Amini SM. Gold nanostructures absorption capacities of various energy forms for thermal therapy applications. J Therm Biol 2019;79:81-4.

39. Robinson JT, Perkins FK, Snow ES, et al. Reduced graphene oxide molecular sensors. Nano Lett 2008;8:3137-40.

40. Liu Z, Robinson JT, Sun X, et al. PEGylated nanographene oxide for delivery of water-insoluble cancer drugs. J Am Chem Soc 2008;130:10876-7.

41. Acik M, Lee G, Mattevi C, et al. Unusual infraredabsorption mechanism in thermally re-duced graphene oxide. Nat Mater 2010;9:840-5.

Cite this article as: Wang $\mathrm{X}$, Li D, Huang X, Luo Q, Li X, Zhang X, Zhang L. A bibliometric analysis and visualization of photothermal therapy on cancer. Transl Cancer Res 2021;10(3):1204-1215. doi: 10.21037/tcr-20-2961 